\title{
Norois
}

Environnement, aménagement, société

$212 \mid 2009 / 3$

Gestion urbaine et inégalités socio-spatiales France, Brésil

\section{Étude morphologique et dynamique des têtes de vallon en milieux forestiers et cultivés : exemple dans le nord du Massif central français}

Head valley study under forest and in cultivated area: example in the northern

French Central Massif

Jean-Pierre Larue

\section{OpenEdition}

Journals

Édition électronique

URL : http://journals.openedition.org/norois/2948

DOI : 10.4000/norois. 2948

ISBN : 978-2-7535-1560-4

ISSN : 1760-8546

Éditeur

Presses universitaires de Rennes

Édition imprimée

Date de publication : 1 décembre 2009

Pagination : 53-68

ISBN : 978-2-7535-1034-0

ISSN : 0029-182X

\section{Référence électronique}

Jean-Pierre Larue, «Étude morphologique et dynamique des têtes de vallon en milieux forestiers et cultivés : exemple dans le nord du Massif central français ", Norois [En ligne], 212 | 2009/3, mis en ligne le 01 décembre 2011, consulté le 10 décembre 2020. URL : http://journals.openedition.org/ norois/2948; DOl : https://doi.org/10.4000/norois.2948 


\title{
ÉTUDE MORPHOLOGIQUE ET DYNAMIQUE DES TÊTES DE VALLON EN MILIEUX FORESTIERS ET CULTIVÉS : EXEMPLE DANS LE NORD DU MASSIF CENTRAL FRANÇAIS
}

\author{
Jean-Pierre Larue \\ GÉodynamique des milieux nAturels et de L'EnVIRonNEMENT \\ (Université de Paris 12-Val de Marne), \\ 61 avenue du Générale-de-Gaulle - 94010 CréteIL cedex \\ larue@univ-paris12.fr
}

\section{RÉSUMÉ}

Des analyses morphométrique et sédimentologique permettent d'étudier la morphologie des têtes de vallon dans le Bocage bourbonnais, région de transition entre le socle du Massif central et la couverture sédimentaire du Bassin parisien, qui juxtapose des espaces forestiers et des milieux cultivés. Le début de l'incision, qui dépend principalement de la pente avant incision et de la surface de l'impluvium, présente des formes différentes selon la lithologie et l'occupation du sol. Des vallons collwviaux en berceau existent entre le début de l'incision et le début de l'écoulement intermittent dans les secteurs cultivés, alors qu'en forêt, l'incision en $V$ et l'écoulement intermittent coüncident le plus souvent. En milieu forestier, les incisions survenues lors de défrichements restent bien conservées et l'érosion continue à se produire après de fortes pluies de saison froide ou à la faveur d'aménagements mal conçus, comme des fossés de drainage acheminant les eaux directement à l'amont des têtes de ravins.

MOTS CLÉS : têtes de vallon - profils longitudinaux - profils transversaux - sédimentologie - forêt - milieu cultivé - Nord du Massif central

\footnotetext{
ABSTRACT

Head valley study under forest and in cultivated area: example in the northern French Central Massif

A morphometrical and sedimentological analysis was carried out as a basis for studying valley heads in the Bocage Bourbonnais, a transition region between the Massif central basement and the Bassin parisien sedimentary cover, which juxtaposes forested and cultivated areas. The beginning of the incision, which is mainly controlled by the gradient before incision and the drainage surface, has different shapes according to lithology and land use. In cultivated areas, shallow dells without any incision take place between the beginning of the incision and the beginning of intermittent flow, whereas under forest, the V shaped incision and the intermittent flow often coincide. Under forest, the incisions caused by old deforestations are well preserved and erosion still occurs after high intensity winter showers or where drainage ditches collect surface runoff directly upstream gully heads.
}

KEY WORDS: head valleys - longitudinal and transverse profiles - sedimentology-evolution under forest and in cultivated area - north Central Massif 
Cet article décrit et essaie d'expliquer les formes des têtes de vallons en milieu forestier et en milieu cultivé. En utilisant l'analyse morphométrique et l'étude des dépôts de remblaiement, il vise à reconstituer les étapes de la morphogenèse de ces incisions. Dans les régions tempérées océaniques de l'Europe du nord-ouest, la morphologie des têtes de vallons diffère beaucoup selon qu'on se trouve en milieu forestier ou en milieu cultivé. Dans le premier cas, les incisions sont fréquemment prononcées et en $\mathrm{V}$ alors que dans le second cas, les vallons ont plutôt une forme en berceau. Lorsque les conditions climatiques et lithologiques sont relativement homogènes, le couvert végétal peut apparaître comme le principal facteur explicatif de cette différence. En protégeant le sol, la forêt limite les processus érosifs sur les versants et favorise l'incision dans les talwegs ou la bonne conservation des incisions préexistantes. En revanche, dans les régions mises en culture, les sols sont moins bien protégés et l'érosion des versants fournit davantage de débris aux talwegs qui se remblaient progressivement lorsque les apports sédimentaires dépassent la capacité de transport des cours d'eau (Bull, 1991; Sklar et Dietrich, 1998; Taylor et Kite, 2006). L'incision peut d'ailleurs reprendre après la reforestation de régions agricoles, ainsi que l'ont montré J. Lach et B. Wyżga (2002) en Pologne. Mais le plus souvent, le moment de l'incision est délicat à cerner, car il varie selon les régions en fonction de l'occupation du sol, de l'évolution des conditions climatiques, mais aussi pour un même cours d'eau, de l'amont à l'aval en raison de la dynamique fluviale (Rommens et al., 2006). Deux groupes d'hypothèses ont été avancés pour les incisions en milieu forestier : soit ces ravins en $\mathrm{V}$ ont une origine climatique, ils se sont alors formés au Tardiglaciaire ou au début de l'Holocène et évoluent très lentement depuis l'installation de la forêt au début de l'Holocène, soit ils ont une origine anthropique, ils se sont formés lors de défrichements et la réinstallation de la forêt a bloqué leur évolution, empêchant leur comblement (Prosser et Soufi, 1998; Lang et al., 2003; Ambers et al., 2006; Lang et Mauz, 2006). Par exemple, dans la forêt de Merdaal, en Belgique centrale, T. Vanwalleghem et al. (2003, 2008) montrent que les ravins en $\mathrm{V}$ ont été façonnés suite à d'anciens défrichements et que leur bonne conservation est due à la reconquête forestière. Mais il ne faut pas oublier le facteur tectonique : les zones en soulèvement sont propices à une forte dissection et à la progression de l'érosion régressive (Menéndez-Duarte et al., 2007; Larue, 2008).

\section{Cadre de l'étude}

L'espace étudié fait partie du Bocage bourbonnais qui est une région de transition entre le socle du Massif central au sud et la couverture sédimentaire du Bassin parisien au nord (fig. 1).

Au nord-ouest du Brethon, les roches cristallines et métamorphiques du socle plongent lentement sous les grès et les argiles du Trias, selon un contact en biseau. Les plateaux ont des altitudes qui décroissent du sud vers le nord : 412 m pour le sommet de l'interfluve situé à l'est de SaintCaprais, 159 m pour le Cher au nord d'Urçay. Cet espace permet d'observer des têtes de vallon, encaissées tantôt dans le socle granitique et métamorphique, tantôt dans les grès et les argiles triasiques de la couverture sédimentaire, et évoluant soit en milieu forestier, soit en milieu cultivé (prairies sur le socle et polyculture sur la couverture sédimentaire). En effet, la forêt de Tronçais, qui est une forêt domaniale depuis 1527, couvre une surface de 10583 ha suffisamment morcelée pour multiplier les contacts forêt-zone agricole le long de plusieurs petits cours d'eau affluents du Cher, de l'Aumance et de la Sologne (fig. 1). La région reçoit en moyenne $790 \mathrm{~mm}$ de précipitations, dont $404 \mathrm{~mm}$ en période de végétation; la température moyenne est de $10,5^{\circ} \mathrm{C}$. Les rivières ont des régimes pluvio-évaporaux océaniques avec de hautes eaux l'hiver et de basses eaux l'été. Par exemple, la Sologne à Ainay-le-Château $\left(88,9 \mathrm{~km}^{2}\right.$ de bassin-versant) a un module de $0,614 \mathrm{~m}^{3} / \mathrm{s}$, les hautes eaux de février atteignent $1,2 \mathrm{~m}^{3} / \mathrm{s}$ et les basses eaux de septembre $0,12 \mathrm{~m}^{3} / \mathrm{s}$, les crues quinquennales $5,8 \mathrm{~m}^{3} / \mathrm{s}$ et les étiages quinquennaux $0,02 \mathrm{~m}^{3} / \mathrm{s}$ (DIREN Centre). Avec un module spécifique de $6,9 \mathrm{l} / \mathrm{s} / \mathrm{km}^{2}$, la Sologne évacue $219 \mathrm{~mm}$ par an, soit un coefficient d'écoulement de $28 \%$. Les lits sont de type mixte, marqués par l'alternance d'affleurement du substratum et de plages d'alluvions, cela peut traduire un déficit sédimentaire holocène (Jacob et al., 2006) ou 


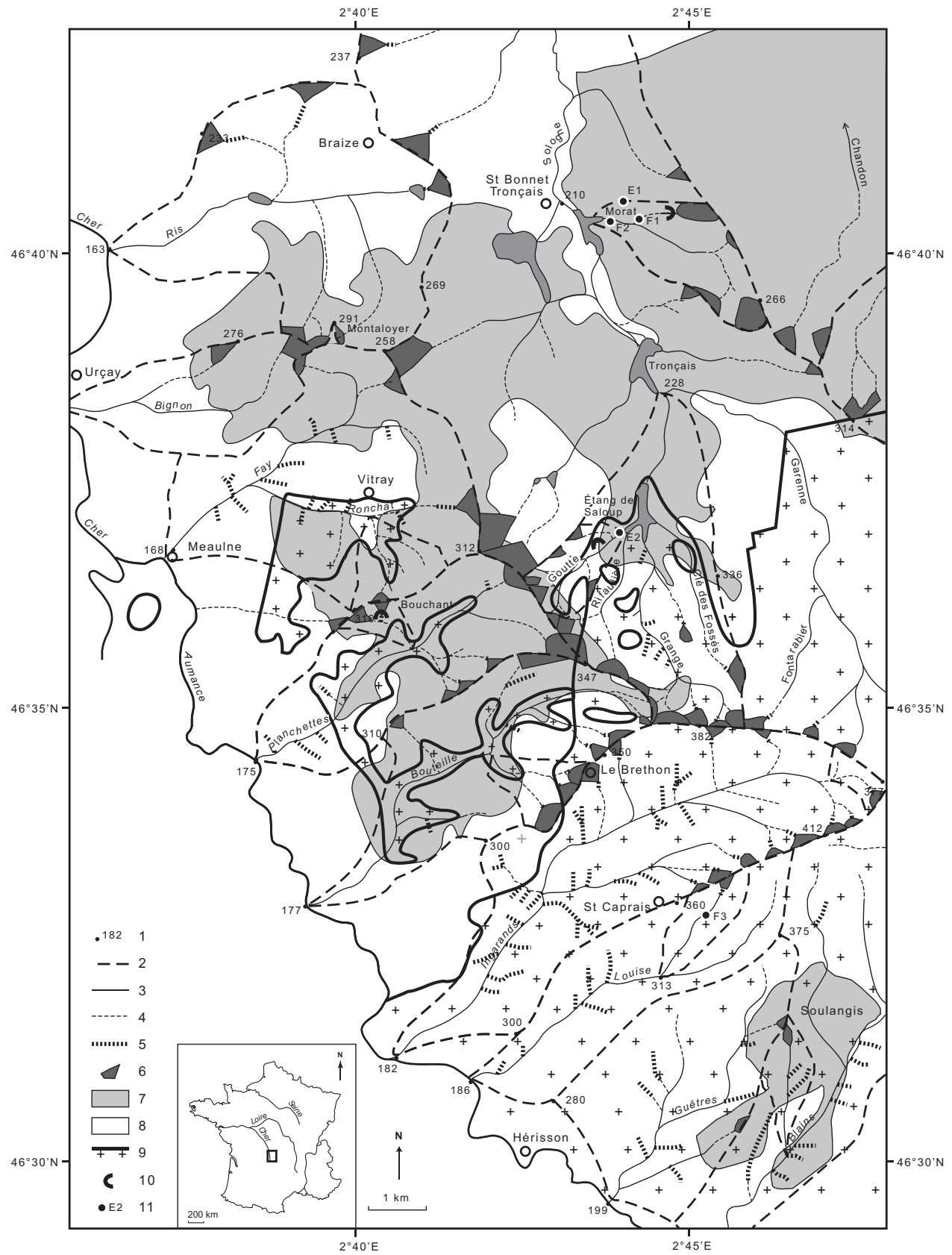

Figure 1 : Carte de localisation

1 : altitude en mètres; 2 : limite de bassin-versant; 3 : écoulement pérenne; 4 : écoulement intermittent ; 5 : vallon sec; 6 : impluvium avant incision; 7 : espace forestier; 8 : prairies et cultures ; 9 : limite socle-couverture sédimentaire; 10 : tête de ravin; 11 : sondages et échantillons.

\section{Location map}

1: altitude in meters; 2: drainage divide; 3: permanent stream; 4: intermittent stream; 5: dry valley; 6: surface drainage before incision; 7: forested area; 8: cultivated area; 9: basement-sedimentary cover limit; 10: gully head; 11: drillings and samples. 
JeAn-Pierre Larue

indiquer que la compétence des cours d'eau est suffisante pour évacuer les alluvions et creuser dans le substrat (Simon et Rinaldi, 2006).

\section{Méthodes}

Après avoir délimité les différents bassins versants, nous avons mesuré la densité de drainage en distinguant les drains à écoulement pérenne, ceux à écoulement intermittent et les vallons secs. Les profils longitudinaux des vallons et des interfluves ont été construits à partir des cartes topographiques IGN (Institut Géographique National) au 1/25000, en utilisant les points cotés et pour les drains l'altitude de chaque isohypse recoupant le talweg. L'équidistance des courbes de $5 \mathrm{~m}$ permet d'obtenir des profils suffisamment précis pour déceler les variations de pente et les principales ruptures de pente. Le calcul des indices SL (longueur-pente) de E. A. Keller et N. Pinter (1996) : $\mathrm{SL}=(\Delta \mathrm{H} / \Delta \mathrm{L})$. L où $\Delta \mathrm{H} / \Delta \mathrm{L}$ est la pente du segment considéré et $\mathrm{L}$ la longueur du chenal en amont du point à partir duquel l'indice est calculé, permet de souligner les moindres ruptures de pente. La forte amplitude des valeurs permet de caractériser tous les changements de pente le long des profils longitudinaux; néanmoins, il faut savoir que pour des secteurs de pente identique, les valeurs augmentent avec la distance des sources. Le graphique adimensionnel $\mathrm{H} / \mathrm{Ho}$ (ratio des altitudes) et L/Lo (ratio des longueurs) (fig. 3, L) permet de superposer et ainsi de comparer les profils de drains de longueur différente (Demoulin, 1998); H est l'altitude du cours d'eau au point mesuré, Ho est la dénivellation entre la source et l'exutoire, L est la distance à l'exutoire du point mesuré et Lo est la longueur du cours d'eau. Pour comparer les profils transversaux des vallons, nous avons utilisé les graphiques adimensionnels H/Ho (ratio des altitudes) et L/Lo (ratio des distances) (Sinha-Roy, 2001); H est l'altitude du versant au point mesuré, Ho est la dénivellation entre l'interfluve le plus élevé et le cours d'eau, L est la distance du point mesuré depuis cet interfluve et Lo est la largeur du vallon, entre les deux interfluves.

Pour chaque tête de vallon, nous avons repéré le début de l'incision et de l'écoulement intermittent. Le début de l'incision est marqué par une rupture de pente séparant, à l'amont une forte pente souvent convexe soumise au ruissellement diffus et à l'aval une pente plus faible en général concave parcourue par un écoulement concentré pérenne ou intermittent. Ce dernier est matérialisé par un lit fluvial tapissé de dépôts alluviaux et presqu'entièrement dépourvu de végétation en raison d'un fonctionnement fluviatile épisodique. Selon les auteurs, l'incision se produit à une certaine distance de la ligne de partage des eaux (Horton, 1945), après une aire critique (Schumm, 1956), après un seuil d'érosion qui dépend de la pente, de l'épaisseur du flot et du type d'écoulement (Montgomery et Dietrich, 1994), mais aussi de la lithologie (Dietrich et Dunne, 1993), là où le profil passe de la convexité à la concavité (Mosley, 1974). D.R. Montgomery et W.E. Dietrich (1988) montrent que dans les milieux tempérés humides, le début de l'incision dépend de l'aire drainée et de la pente en amont de l'incision. Aussi, pour chaque début d'incision, nous avons mesuré la distance entre la ligne de partage des eaux et le début de l'incision et de l'écoulement intermittent, la pente et la surface drainée avant l'incision et avant l'écoulement intermittent. La lithologie et le couvert végétal ont également été relevés. Les relations entre ces données ont été analysées.

Malgré le peu d'affleurements dans les vallons, nous avons, à l'aide de sondages à la tarière, essayé de localiser les zones d'accumulation alluviale et d'évaluer leur épaisseur. Des analyses granulométriques aident à comprendre l'origine et les modes de mise en place de ces dépôts de fond de vallon. La granulométrie des dépôts a été utilisée par E. Smolska (2007) pour distinguer ceux d'origine climatique de ceux accumulés suite à des défrichements. Les sédiments provenant de l'érosion des sols cultivés sur les versants sont plus fins et plus riches en humus que ceux issus de la formation de ravins dans les fonds de vallon. Les granulométries ont été effectuées avec le granulomètre laser LS Coulter qui permet de classer les particules comprises entre $0,375 \mu \mathrm{m}$ et $2000 \mu \mathrm{m}$, ce qui couvre la totalité des fractions sableuses, limoneuses et argileuses (Blott et al., 2004). Concentrés selon la méthode densimétrique de séparation au bromoforme (Parfenoff et 
al., 1970), les minéraux lourds transparents, de densité > 2,89, ont été déterminés par R. Étienne au microscope optique. Les pourcentages de minéraux transparents ont été calculés à partir de l'analyse d'environ 200 grains. Les associations de minéraux lourds renseignent sur l'origine des flux, mais dépendent aussi de l'altération du matériau (Larue et Étienne, 1998).

\section{Analyse morphométrique}

La densité des drains varie entre $1,54 \mathrm{~km} / \mathrm{km}^{2}$ dans le bassin du Ronchat et 2,95 km/ $\mathrm{km}^{2}$ dans celui des Ingarands. Elle est toujours plus forte pour les bassins versants s'étendant sur le socle (moyenne $=2$ ) que pour ceux développés dans la couverture triasique (moyenne $=1,6$ ). L'écoulement pérenne est plus important sur le socle (50\%) que sur la couverture sédimentaire (38\%); le pourcentage d'assèchement l'emporte dans le socle (32 \% des drains du bassin des Ingarands) et l'écoulement intermittent arrive en tête dans les bassins de la couverture ( $58 \%$ des drains dans le bassin des Planchettes).

Les mesures réalisées sur 81 têtes de vallon montrent que la localisation de l'incision dépend de plusieurs facteurs. La distance de la ligne de partage des eaux et la surface de l'impluvium avant incision diminuent quand la pente avant incision augmente, mais on enregistre de grandes différences selon la lithologie et le couvert végétal (fig. 2).

On peut observer que la relation pente avant incision-impluvium est meilleure que celle entre pente avant écoulement intermittent et impluvium (fig. 2, A, B). Les relations entre impluvium et pente avant incision et avant écoulement intermittent se révèlent meilleures en milieu forestier qu'en secteur cultivé, elles sont particulièrement médiocres en milieu cultivé sur la couverture triasique (fig. 2, J, L). La surface de l'impluvium est mieux corrélée avec la pente avant incision (fig. 2, B) que ne l'est la distance de la ligne de partage des eaux, excepté pour les vallons cultivés sur la couverture triasique (fig. 2, J). Pour la région étudiée, la surface moyenne de l’impluvium avant incision augmente du sud au nord avec la diminution de l'énergie du relief. Elle est aussi plus petite sous forêt qu'en milieu cultivé : respectivement 12,9 ha et 28 ha sur la couverture sédimentaire, et 8,5 ha et 13 ha sur le socle. En milieu forestier, le début de l'incision correspond le plus souvent au début de l'écoulement intermittent, alors qu'en milieu cultivé, un petit vallon sec, en berceau et tapissé de colluvions, s’intercale entre les deux points.

Les têtes de vallon présentent plusieurs types de forme. En général, sur le premier kilomètre à partir de la ligne de partage des eaux, on distingue dans l'axe du talweg les sections suivantes : un plan incliné sans incision formant l'impluvium, un secteur à plus forte pente où l'incision s'accentue, un secteur élargi tapissé d'alluvions et de colluvions où la pente se ralentit, une rupture de pente correspondant à une forte incision dans la roche en place, puis un nouveau ralentissement de pente avec dépôts d'alluvions. En milieu forestier, le plus souvent, l'incision en $V$ augmente progressivement vers l'aval, sans rupture de pente, mais parfois, comme dans les vallons de Morat ou de la Goutte de l'étang de Saloup, des ruptures de pente avec cascades témoignent d'une érosion régressive efficace (photos 1 et 2 ).

Dans le bassin du Bouchant, nous avons observé un ravin affluent du vallon principal, qui n'est plus fonctionnel aujourd'hui : des arbres centenaires poussent dans le fond qui présente une forme en $\mathrm{V}$ du fait de l'adoucissement des bords par l'érosion (photo 3). En milieu cultivé, le début de l'incision correspond à un brutal ralentissement de la pente et se prolonge par un ample vallon en berceau dépourvu d'écoulement sur une courte distance (50 à 100 m), puis l'écoulement intermittent ou pérenne produit un petit chenal légèrement encaissé dans la forme en berceau (photo 4).

Les profils longitudinaux des talwegs et des interfluves (fig. 3) s'écartent progressivement vers l'aval, indiquant que l'incision augmente vers l'aval. 


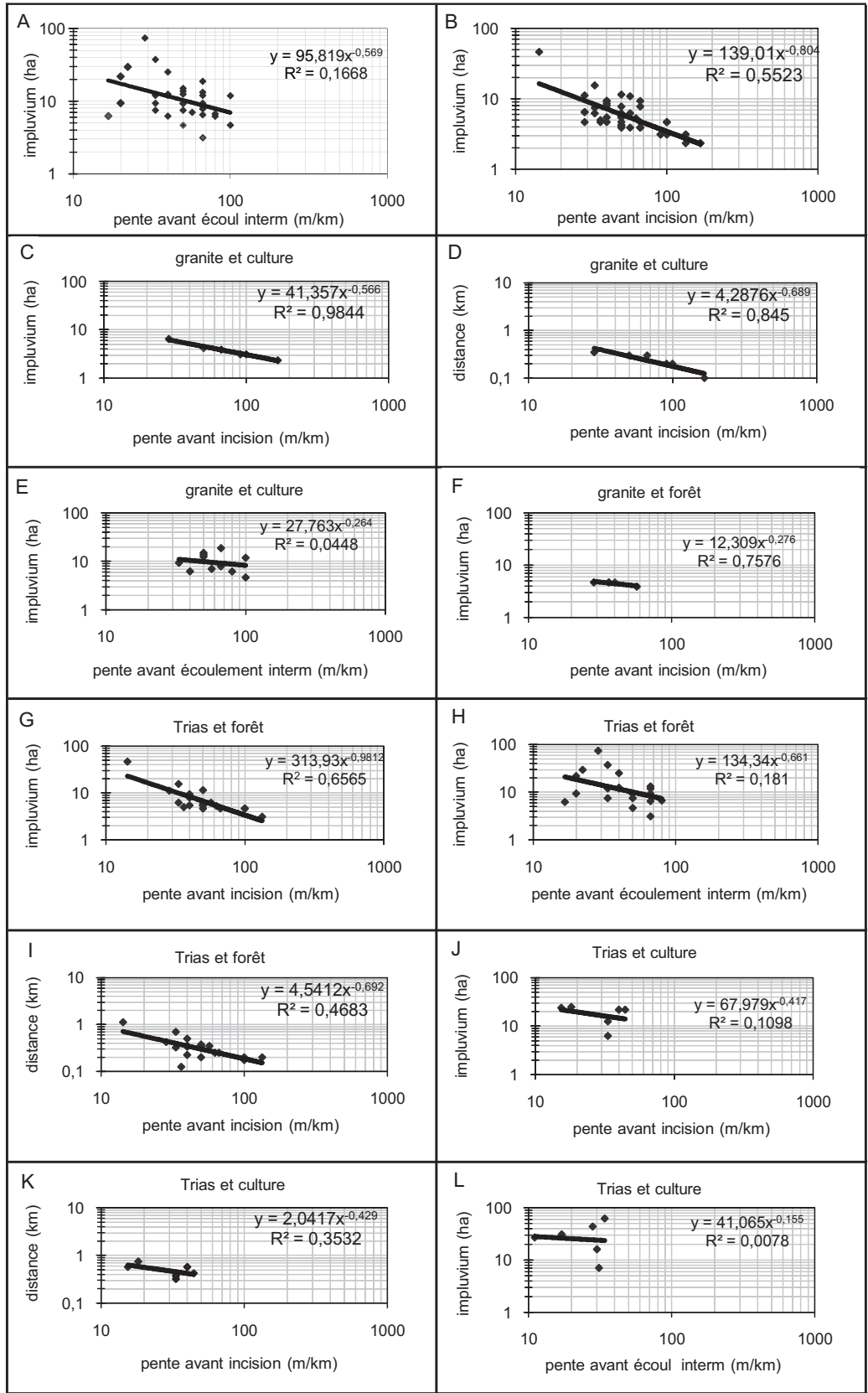

Figure 2 : Relations entre pente avant écoulement intermittent et impluvium, entre pente avant incision et impluvium, entre pente avant incision et distance de la ligne de partage des eaux, pour tous les vallons (A, B), pour les vallons granitiques cultivés $(\mathrm{C}, \mathrm{D}, \mathrm{E})$ et sous forêt $(\mathrm{F})$ pour les vallons de la couverture sédimentaire cultivés $(\mathrm{J}$, K, $\mathrm{L})$ et sous forêt $(\mathrm{G}, \mathrm{H}, \mathrm{I})$.

Relationships between gradient before intermittent flow and drainage surface, between gradient before incision and drainage surface, between gradient before incision and drainage divide distance, for all the dells $(A, B)$, for the dells in the basement in cultivated area $(C, D, E)$ and under forest $(F)$, for the dells in the sedimentary cover in cultivated area $(J, K, L)$ and under forest $(G, H, I)$. 


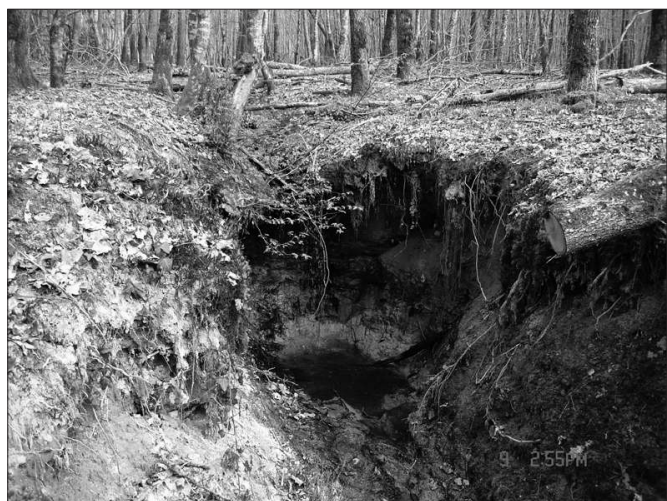

Photo 1 : Ravin à l'amont du vallon de Morat (9 avril 2007)

Gully head in the Morat catchment

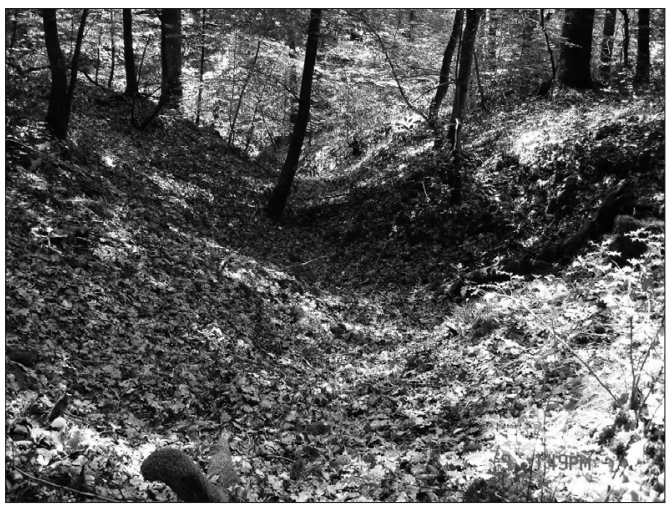

Photo 3 : Ravin affluent du vallon du Bouchant (3 juin 2008)

Tributary gully of the Bouchant dell

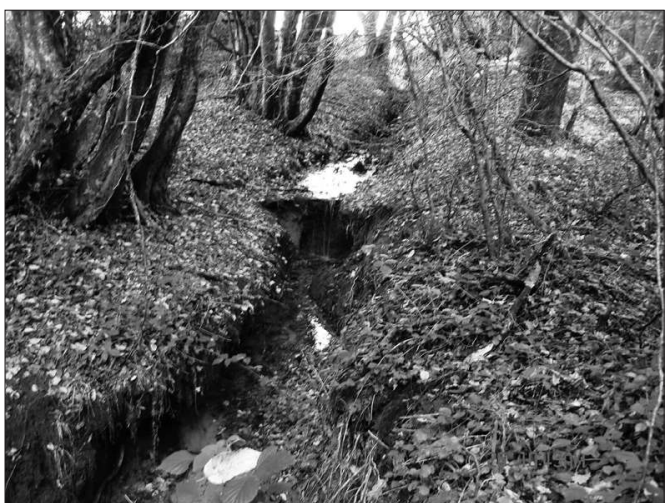

Photo 2 : Rupture de pente et cascade sur un affluent du Goutte de l'étang de Saloup (26 octobre 2008)

Knickpoint and waterfall on the Goutte de l'étang de Saloup tributary

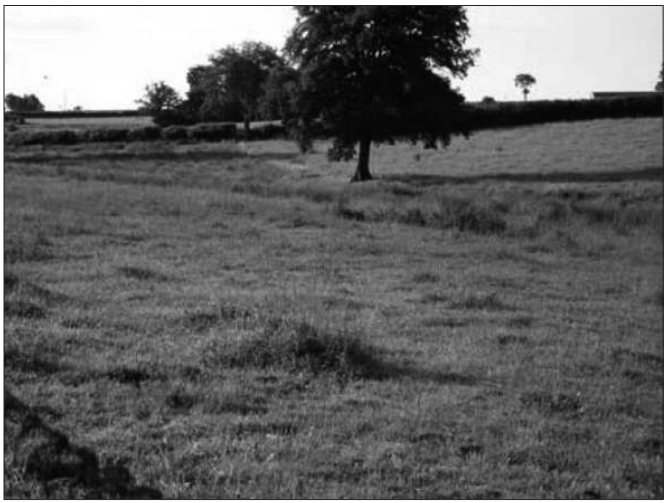

Photo 4 : Vallon en berceau des Gozards (21 juin 2008) out any incision

The Gozards shallow dell valley head with-

Les indices SL et les pentes donnent des pics qui se localisent surtout vers l'aval, sauf pour le Ronchat (fig. 3, C) et le vallon des Gozards (fig. 3, F). Le graphique adimensionnel H/Ho-L/Lo (fig. 3, L) révèle des profils irréguliers à faible concavité, excepté pour le vallon des Gozards. Le profil du Bouchant présente une convexité aval (fig. 3, A) et celui du Brethon une convexité amont (fig. 3, E). La pente avant l'incision est très variable, mais elle est plus forte en milieu cultivé sur le socle qu'en milieu forestier sur la couverture triasique : respectivement $60 \%$ au nord et à l'est du Brethon et 18,7 \%o pour le vallon de Morat. Par exemple, sur les flancs du Point de Vue qui culmine à $412 \mathrm{~m}$, les têtes de vallons secs débutent vers $375 \mathrm{~m}$ d'altitude et se poursuivent par des incisions peu marquées présentant des pentes longitudinales fortes, environ $80 \%$, jusqu'aux sources amorçant un écoulement pérenne à partir de $365 \mathrm{~m}$, dans un vallon ample marécageux à pente plus faible. En localisant les incisions sur le profil longitudinal, on constate qu'elles se trouvent toutes immédiatement à l'aval de la première rupture de pente enregistrée à partir des sources. Mais la forme varie selon qu'on se trouve en milieu cultivé ou en milieu forestier : on a d'amples vallons marécageux dans le premier et des incisions en $\mathrm{V}$ fonctionnelles dans le second. 


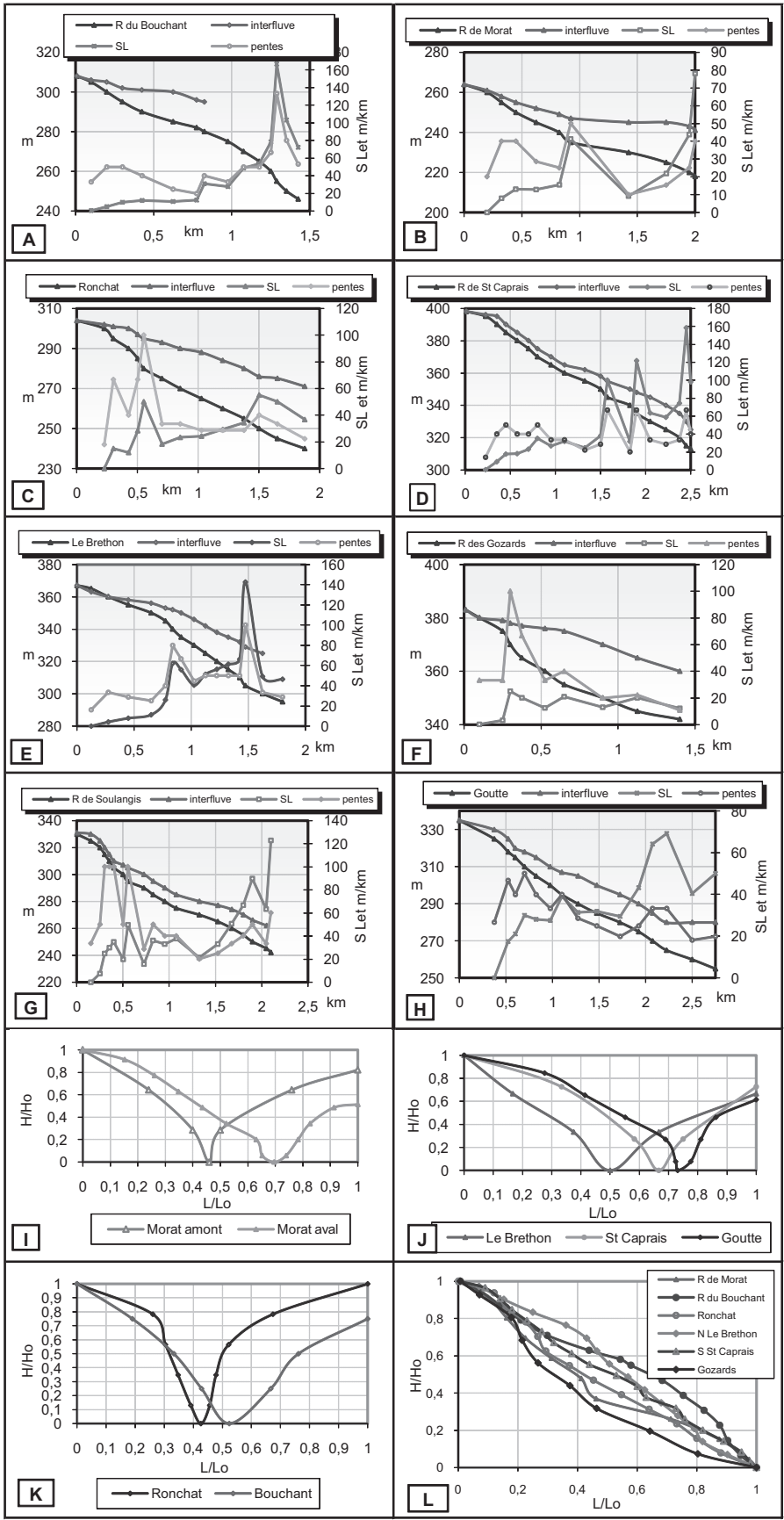

Figure 3 : A à H : Profils longitudinaux des talwegs et des interfluves, pentes et SL indices (Keller et Pinter, 1996); I à K : Profils transversaux des vallons (graphiques adimensionnels $\mathrm{H} / \mathrm{Ho}$ [ratio des altitudes] et $\mathrm{L} / \mathrm{Lo}$ [ratio des distances]); L : Profils longitudinaux comparés (graphiques adimensionnels H/Ho [ratio des altitudes] et L/Lo [ratio des distances]).

A to H : Longitudinal profiles, gradients, SL indexes (Keller and Pinter, 1996) and interfluve profiles; I to K : Transverse profiles: dimensionless curves H/Ho-L/Lo (ratio of altitude-ratio of distance); L: Longitudinal profiles: dimensionless curves $\mathrm{H} / \mathrm{Ho}-\mathrm{L} / \mathrm{Lo}$ (ratio of altitude-ratio of distance). 
Les profils transversaux normalisés (fig. 3, I, J, K) montrent que l'incision représente entre 22 et $35 \%$ de la section considérée. Elle est plus forte dans le socle que dans le Trias, car les vallons sont plus amples dans le premier. Les pentes augmentent depuis l'interfluve jusqu'au cours d'eau, ce qui témoigne de formes récentes : le creusement vertical a été plus rapide que l'évolution des versants.

Le vallon de la Goutte de l'étang de Saloup (fig. 3H) présente les formes les plus variées, car il se développe tantôt en milieu forestier, tantôt en milieu cultivé. Le petit cours d'eau de 2,75 km de longueur qui l'a façonné coule presque entièrement dans le granite de Tronçais, granite leucocrate à gros grains, à biotite, muscovite et sillimanite (Turland et al., 1990). La pente moyenne atteint $29 \%$, le profil longitudinal présente une faible concavité et deux ruptures de pente situées dans la zone de contact forêt-cultures (fig. 1). La première se trouve à la sortie du secteur boisé amont et la seconde à l'entrée dans le massif forestier entourant l'étang de Saloup, elles ont des pentes atteignant respectivement $40 \%$ et $33 \%$. L'encaissement du vallon augmente progressivement vers l'aval : $10 \mathrm{~m}$ avant la première rupture de pente, $15 \mathrm{~m}$ à l'aval de cette dernière et $25 \mathrm{~m}$ au niveau de l'exutoire formé par l'étang de Saloup. En milieu forestier, les versants sont abrupts et convexes, la pente augmente vers le bas en se rapprochant du lit actuel : $20 \%$ près de l'interfluve, $400 \%$ à la base du versant. En milieu cultivé, les versants plus doux deviennent concaves et le fond du vallon présente une forme en berceau. Le lit mineur apparaît plus large et plus encaissé en milieu forestier qu'en milieu cultivé. Dans le secteur médian forestier, sa largeur atteint $80 \mathrm{~cm}$ et la profondeur varie entre 40 et $100 \mathrm{~cm}$, le fond plat est encombré de bancs sableux et les berges présentent des abrupts et des surplombs bien développés dans les rives concaves des méandres encaissés. Les berges sont modelées dans des sables fluviatiles à stratification inclinée vers l'aval et le cours d'eau incise légèrement le substrat granitique. Le lit majeur présente une topographie très accidentée, avec des dépressions allongées et arquées suggérant d'anciens méandres aujourd'hui perchés ( $1 \mathrm{~m}$ au dessus du lit mineur). Dans le secteur de la deuxième rupture de pente, on observe des ravins affluents soulignant la forte activité de l'érosion régressive actuelle : la photo 2 montre l'abrupt de tête de $90 \mathrm{~cm}$ de hauteur et la petite cascade qui en résulte.

\section{Les dépôts de remblaiement}

Les formes d'érosion l'emportent largement sur celles d'accumulation. Les dépôts alluviaux et colluviaux ne sont conservés que dans les vallons supérieurs pour les secteurs cultivés et immédiatement à l'aval des incisions de têtes de vallons dans les zones boisées. Les vallons en berceau, fréquents sur le socle, présentent un remplissage peu épais, formé de sables graveleux (30

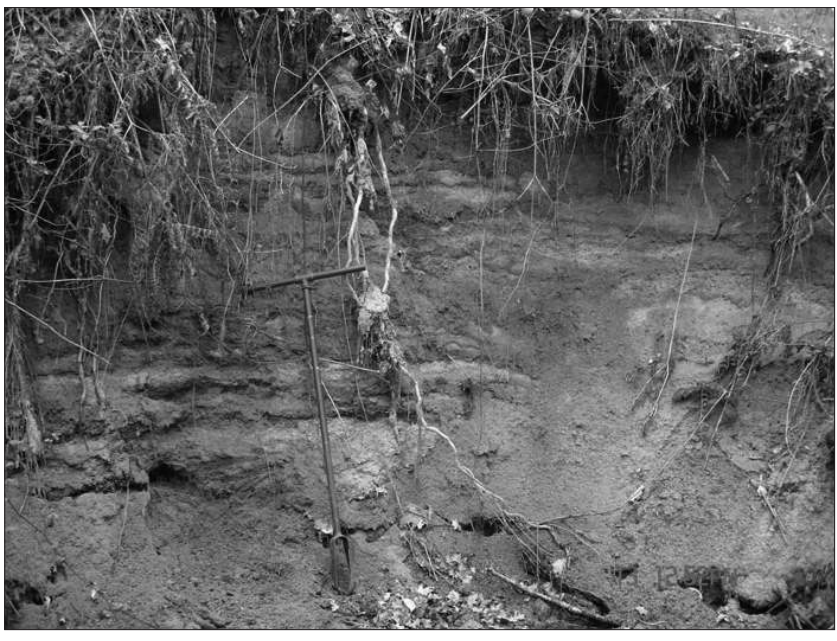

à $40 \mathrm{~cm}$ d'épaisseur) surmontés de dépôts organo-minéraux excédant rarement un mètre de puissance. Lorsqu'ils sont conservés, les remblaiements localisés à l'aval des ravinements sont plus épais (jusqu’à 2,50 m dans le vallon de la Goutte de l'étang de Saloup) et principalement sableux (photo 5).

Photo 5 : Berge du ruisseau de la Goutte de l'étang de Saloup montrant la lithostratigraphie du remblaiement alluvial (13 avril 2009)

Infilling deposits of the Goutte de l'étang de Saloup brook 
Au sud de l'étang de Saloup, les dépôts de berge du ruisseau révèlent des sables ocres disposés en stratification inclinée et incluant des charbons de bois à 2,20 m de profondeur (photo 5). La granulométrie de l'échantillon E2 indique des sables fluviatiles moyens à grossiers (médiane $=370 \mu \mathrm{m}$ ) et moyennement classés (So Trask $=1,6$ ), transportés par saltation et roulage.

Dans le vallon du Rond de Morat, les sondages effectués à l'aval de l'incision (F1) et près de l'étang de Morat (F2) montrent des sédiments fluviatiles sableux dont la granulométrie décroît du bas vers le haut et de l'amont à l'aval (tabl. 1).

\begin{tabular}{|c|c|c|c|c|c|c|}
\hline $\begin{array}{l}\text { Ech. F 1, } \\
\text { R de Morat }\end{array}$ & $\begin{array}{l}\text { Médiane } \\
(\mu \mathrm{m})\end{array}$ & $\begin{array}{c}\text { Argiles } \\
(\%<2 \mu \mathrm{m})\end{array}$ & $\begin{array}{l}\text { Limons }(\% \\
\text { entre } 2 \text { et } 50 \\
\mu \mathrm{m})\end{array}$ & $\begin{array}{l}\text { Sables }(\%< \\
50 \mu \mathrm{m})\end{array}$ & So (Trask) & As (Trask) \\
\hline $0,40 \mathrm{~m}$ & 185 & 1,41 & 15,5 & 83,1 & 2,23 & 0,86 \\
\hline $0,60 \mathrm{~m}$ & 407 & 0,74 & 7,5 & 91,7 & 1,95 & 0,70 \\
\hline $0,80 \mathrm{~m}$ & 741 & 0,55 & 4,6 & 94,8 & 1,62 & 0,81 \\
\hline $1 \mathrm{~m}$ & 557 & 0,58 & 5,5 & 93,9 & 1,62 & 0,84 \\
\hline & & & & & & \\
\hline $\begin{array}{l}\text { Ech. F } \\
\text { 2, Étang de } \\
\text { Morat }\end{array}$ & $\begin{array}{l}\text { Médiane } \\
(\mu \mathrm{m})\end{array}$ & $\begin{array}{c}\text { Argiles } \\
(\%<2 \mu \mathrm{m})\end{array}$ & $\begin{array}{l}\text { Limons }(\% \\
\text { entre } 2 \text { et } 50 \\
\mu \mathrm{m})\end{array}$ & $\begin{array}{l}\text { Sables }(\%< \\
50 \mu \mathrm{m})\end{array}$ & So (Trask) & As (Trask) \\
\hline $0,40 \mathrm{~m}$ & 247 & 0,85 & 7,3 & 91,8 & 1,96 & 1,02 \\
\hline $0,60 \mathrm{~m}$ & 261 & 0,81 & 5,6 & 93,6 & 1,63 & 0,92 \\
\hline $0,80 \mathrm{~m}$ & 363 & 0,52 & 2,9 & 96,6 & 1,66 & 1,02 \\
\hline $\begin{array}{c}\text { Ech. F 3, } \\
\text { Vallon Saint } \\
\text { Caprais }\end{array}$ & $\begin{array}{l}\text { Médiane } \\
(\mu \mathrm{m})\end{array}$ & $\begin{array}{c}\text { Argiles } \\
(\%<2 \mu \mathrm{m})\end{array}$ & $\begin{array}{l}\text { Limons }(\% \\
\text { entre } 2 \text { et } 50 \\
\mu \mathrm{m})\end{array}$ & $\begin{array}{l}\text { Sables }(\%< \\
50 \mu \mathrm{m})\end{array}$ & So (Trask) & As (Trask) \\
\hline $0,40 \mathrm{~m}$ & 520 & 0,62 & 8 & 91,4 & 2,02 & 0,75 \\
\hline $0,80 \mathrm{~m}$ & 641 & 0,48 & 5 & 94,5 & 1,76 & 0,80 \\
\hline
\end{tabular}

Tableau 1 : Caractéristiques granulométriques des dépôts de remblaiement (granulométries effectuées par M. Bouabdallah)

As : indice d'asymétrie de Trask $=\left(\mathrm{Q}^{*}{ }^{*} \mathrm{Q} 3 / \mathrm{Md}^{2}\right)$; So : indice de classement de Trask $=(\sqrt{ } \mathrm{Q} 3 / \mathrm{Q} 1)$.

Grain-size characteristics of the infilling deposits (grain sizes made by M. Bouabdallah)

As: Trask asymmetry index $\left(\mathrm{Q} 1{ }^{*} \mathrm{Q} 3 / M d^{2}\right)$; So: Trask sorting index $(\sqrt{ } 33 / \underline{Q} 1)$.

Sur le plan vertical, la médiane passe de $741 \mu \mathrm{m}$ à $0,80 \mathrm{~m}$ de profondeur à $407 \mu \mathrm{m}$ à $0,40 \mathrm{~m}$ de profondeur, immédiatement à l'aval de l'incision, et de $363 \mu \mathrm{m}$ à $247 \mu \mathrm{m}$ pour les mêmes profondeurs près de l'étang de Morat. Les dépôts sont mieux classés à la base (So Trask = 1,6) que dans la partie supérieure (So Trask $=2,2$ ) et l'asymétrie se fait vers les petites tailles. Vers l'aval, l'asymétrie s'estompe et s'oriente vers les éléments grossiers, indiquant que les fractions fines sont exportées vers le cours principal, ici la Sologne. Les sables inférieurs, qui contiennent aussi quelques fragments de charbon de bois, ont une composition en minéraux lourds semblable à celle des sables pliocènes couronnant les interfluves, alors que les sables supérieurs sont plus pauvres en espèces probablement en raison de l'altération liée à la pédogenèse (tabl. 2).

Dans le sondage du vallon de Saint-Caprais, on retrouve la même évolution du bas vers le haut, mais les dépôts apparaissent plus grossiers (520 $\mu \mathrm{m}$ dans la partie supérieure) (tabl. 1). Le matériau provient essentiellement des arènes granitiques. 


\begin{tabular}{|c|c|c|c|c|c|c|c|c|c|c|}
\hline 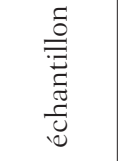 & 气̃ & 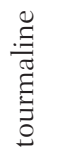 & $\stackrel{\stackrel{\vartheta}{\Xi}}{\underset{Z}{Z}}$ & $\stackrel{\mathscr{\Xi}}{\stackrel{\Xi}{\Xi}}$ & 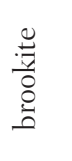 & $\begin{array}{l}\vec{E} \\
\vec{d} \\
\vec{d}\end{array}$ & 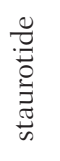 & 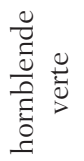 & 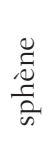 & 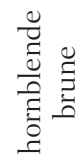 \\
\hline $\begin{array}{c}\mathrm{F} 1, \\
0,40 \mathrm{~m}\end{array}$ & 74 & 25 & & & & 1 & & & & 1 \\
\hline $\begin{array}{c}\mathrm{Fl}, \\
0,80 \mathrm{~m}\end{array}$ & 71 & 23 & 1 & & 0,5 & 2,5 & & & 1 & 1,2 \\
\hline E1 & 64 & 27 & 2 & 0,5 & 0,5 & 4 & 0,5 & 0,5 & & 1 \\
\hline
\end{tabular}

Tableau 2 : Composition en minéraux lourds (déterminations R. Étienne) Heavy minerals composition (R. Étienne determinations)

Les résultats obtenus suggèrent que les matériaux inférieurs plus grossiers proviennent des incisions dans le substrat alors que les sables plus argileux de la partie supérieure sont issus de l'érosion diffuse des sols sur les versants.

\section{Évolution pléistocène, holocène et historique des têtes de vallons}

\section{ÉVOLUTION PLÉISTOCÈNE}

L'irrégularité des profils longitudinaux des vallons supérieurs, dans leur premier kilomètre depuis la ligne de partage des eaux, peut s'expliquer par l'évolution enregistrée au cours du Pléistocène. Du début de l'incision des cours d'eau à aujourd'hui, les interfluves n'ont subi qu'une très faible érosion, ainsi qu'en témoigne la conservation de placages pliocènes, alors que les vallées ont été fortement creusées $(80$ à $100 \mathrm{~m}$ ) : il en résulte une augmentation progressive du dénivelé entre les interfluves et les exutoires des petits cours d'eau, et donc de la pente globale de ces derniers. En roche dure, la plupart des cours d'eau ne sont pas parvenus à régulariser leur profil longitudinal (forme concave), d'autant plus que les périodes de creusement ont été limitées aux périodes de transition entre glaciaire et interglaciaire (Bridgland et al., 2004). Les knickpoints, bien signalés par les indices SL et les pentes (fig. 3), se sont, non seulement maintenus, mais accentués au cours du creusement quaternaire saccadé; cela s'accorde avec les analyses récentes de J. D. Phillips et J. D. Lutz (2008). Le soulèvement quaternaire du horst de Hérisson-Forêt de Tronçais explique sa forte dissection et l'ampleur de l'encaissement des affluents de l'Aumance (Larue, 2008). Mais là où le soulèvement a été trop rapide pour que l'érosion régressive puisse remonter jusqu'à l'amont, l'incision s'est trouvée bloquée dans les roches résistantes du socle et des vallées amples en berceau subsistent à l'amont, comme dans les alvéoles granitiques des vallons des Gozards ou de Saint-Caprais (photo 4). Les dépôts conservés ressemblent beaucoup à ceux étudiés sur les bas plateaux limousins par B. Allée et al. (1997), B. Allée (2003), B. Allée et L. Lespez (2006) qui signalent des alluvions périglaciaires peu épaisses surmontées par un remblaiement organo-minéral épais qui débute avec l'anthropisation du milieu.

\section{INCISION TARDIGLACIAIRE}

L'encaissement actuel des vallons en $V$ est pour l'essentiel acquis lors du creusement tardiglaciaire effectué par des cours d'eau plus puissants que les ruisseaux actuels et capables de sculpter localement des méandres encaissés, comme ceux observés dans le vallon de la Goutte de l'étang de Saloup. Le déblaiement total des alluvions périglaciaires atteste de l'efficacité du creusement tardiglaciaire enregistré dans la plupart des vallées du Bassin parisien et du Massif central (Raynal, 
1984 ; Pastre et al., 1997; Larue, 1999a; Cubizolle et al., 2001). Cette forte incision résulte de l'augmentation des précipitations et des débits associée au déclin de la fourniture sédimentaire liée à l'arrêt de la cryoclastie (Pastre et al., 2003). Les différences entre bassins versants peuvent s'expliquer par les conditions locales comme l'a montré K. N. Wilkinson (2003) pour les vallons secs du sud de l'Angleterre. Les matériaux issus de l'incision tardiglaciaire ne se retrouvent pas dans les têtes de vallon car ils ont été exportés vers l'aval.

\section{ÉVOLUTION HISTORIQUE}

Le couvert végétal semble aussi jouer un rôle important. Si les corrélations pente-impluvium sont meilleures sous forêt qu'en milieu cultivé pour la couverture sédimentaire, c'est parce que l'incision est mieux conservée dans le premier cas que dans le second. En fixant et en protégeant les sols sur les pentes, la végétation forestière permet le maintien des versants abrupts de l'incision; les écoulements intermittents qui se produisent au fond de l'incision sont constitués d'eaux relativement claires et suffisamment érosives pour entretenir la forme en $\mathrm{V}$. En revanche, la mise en culture supprime tout ou partie de cette protection et l'érosion diffuse finit par combler tout ou partie de l'incision. Aussi, l'impluvium est-il plus petit sous forêt qu'en zone cultivée. Les ruptures de pente avec cascades et les ravins signalés sont tous situés à l'aval d'impluviums forestiers anormalement petits. La présence de fossés de drainage semble expliquer cette situation : ces fossés concentrent les eaux de ruissellement peu chargés qui acquièrent ainsi une forte capacité érosive. Ces formes récentes dues à l'érosion régressive se rencontrent aussi bien dans la couverture sédimentaire (vallon de Morat) que dans le socle (vallon de la Goutte de l'étang de Saloup). Dans le fond de ce dernier, la formation de terrasses sableuses est due à l'incision qui a pu être provoquée par la reforestation du bassin amont. En effet, la reforestation des bassins supérieurs est à l'origine de l'incision et du rétrécissement des lits fluviaux dans les Alpes du sud (Liébault et Piégay, 2002; Liébault et al., 2005). Par ailleurs, comme l'a montré M. Mc Bride et al. (2008) dans le Vermont, les lits mineurs apparaissent aussi plus larges et plus profonds en milieu forestier qu'en milieu enherbé, mais aussi plus larges dans le socle que dans la couverture triasique.

Les incisions de tête de vallon, qu'on trouve en milieu forestier quelle que soit la lithologie, peuvent s'être produites lors de phases de défrichement et de mise en culture ou à la faveur d'incendies d'origine naturelle. Il est donc difficile d'établir une chronologie précise et générale de ces crises érosives dans les têtes de vallon. On retrouve le même phénomène dans la vallée de la Dore où les cônes d'accumulation à la base des versants ont été datés à partir de charbons de bois entre $7970 \pm 60 \mathrm{BP}$ et $220 \pm 50 \mathrm{BP}$ (Cubizolle et al., 2001), mais aussi dans les marais de la Champagne berrichonne où des charbons de bois, datés entre 8500 et 3300 avant J.-C., témoignent de feux d'origine climatique (Vannière et Laggoun-Defarge, 2002). En forêt de Tronçais, E. Dambrine et al. (2007) signalent 108 établissements gallo-romains dans l'espace forestier actuel et montrent que cette agriculture ancienne a des répercussions sur la composition floristique actuelle. Les zones ayant été cultivées renferment plus d'espèces que celles restées boisées (160 espèces pour les premières contre 131 pour les secondes) et se différencient par la présence de plantes rudérales. On peut constater que les ravins observés sont tous situés à proximité de ces établissements galloromains, ce qui renforce l'explication anthropique de ces formes et s'accorde avec les analyses de T. Vanwalleghem et al. (2008) en forêt de Merdaal. La présence de charbons de bois dans les alluvions localisées à l'aval des incisions de têtes de vallon atteste de brûlis et de défrichements par le feu. Le ravin affluent du vallon du Bouchant n'a pu être creusé qu’à la faveur de défrichement, lorsque les sols sans ou avec très peu de couverture végétale ont été soumis à des précipitations exceptionnelles, comme par exemple celles signalées au Moyen Âge par A. Lang et B. Mauz (2006) et M. Dotterweich (2008) en Europe centrale. L'absence de dépôts conservés à l'aval du ravin peut être expliquée par la forte pente (qui en plus augmente vers l'aval) du vallon du Bouchant qui a facilité l'exportation des matériaux (fig. 3, A). Les alluvions plus grossières trouvées à la base des 
remblaiements proviennent de ravins atteignant le substrat, alors que les dépôts supérieurs plus fins résultent d'une érosion plus diffuse sur les versants.

Bien que la forêt domaniale de Tronçais ait été rattachée au domaine royal en 1527 par la confiscation des biens de Charles III, duc de Bourbon, le couvert végétal a beaucoup varié et les deux périodes de forte dégradation de la forêt (XVI-XVII ${ }^{\mathrm{e}}$ siècles et fin XVIII ${ }^{\mathrm{e}}$-début XIX ${ }^{\mathrm{e}}$ siècle) ont pu permettre le ravinement des secteurs en pente et l'apparition de ravins (Bertrand, 1996; Larue, 1999b; ONF Auvergne). Au XvI ${ }^{\mathrm{e}}$ siècle, la forêt était avant tout un vaste espace nourricier, parcours des bêtes domestiques, réserve de terre en attente de défrichement, espace de chasse et de cueillette. Lorsque Colbert, sous Louis XIV, fait procéder à un état des lieux des grandes forêts royales, le compte rendu de ses conseillers est alarmant. La dégradation de la forêt et le désordre des coupes sont tels que le recépage doit être prescrit sur la presque totalité de la surface forestière. Seuls 150 ha de bonne futaie sont préservés dans l'inaccessible canton de la Bouteille. À la fin du XVIII ${ }^{\mathrm{e}}$ siècle, les caisses royales ont besoin de nouvelles recettes, et tout doit être fait pour permettre le développement des industries récentes. Ainsi en 1779, les futaies de Tronçais, alors âgées de 40 à 100 ans, sont exploitées pour alimenter les forges du Berry, et dès 1788, celles de Tronçais. La forêt est transformée en taillis et en taillis-sous-futaie. Seul le centre du massif est conservé en futaie pour répondre aux futurs besoins de la Marine. Face à cette dégradation, le comte de Buffévent met en place vers 1835, le premier plan de gestion de la forêt de Tronçais qui, pour régénérer les chênes, remplace le rejet de souche par des semis de glands, naturels ou artificiels. Les coupes progressives de régénération vont s'imposer à Tronçais : une coupe d'ensemencement, suivie d'une coupe secondaire, et pour finir une coupe définitive, le tout devant se réaliser sur une dizaine d'années. Par ailleurs, il préconise l'utilisation du pin sylvestre pour coloniser les landes à bruyères et les mauvais terrains hydromorphes. Cette gestion va contribuer à fortement limiter la morphogenèse en forêt de Tronçais. Pourtant localement, la construction de fossés de drainage peut favoriser la concentration des eaux et l'incision brutale sous forme de ravins, comme dans le vallon de Morat ou autour de la butte de Montaloyer.

\section{Conclusion}

La morphologie des vallons se révèle très variée en fonction de la lithologie, mais aussi de l'utilisation du sol actuelle et passée, car le couvert végétal a pu varier plusieurs fois au cours du temps avec l'anthropisation. On trouve souvent un emboîtement et une juxtaposition de plusieurs générations de formes, aussi convient-il de ne pas généraliser à partir de quelques exemples. Le soulèvement quaternaire de la région étudiée explique les fortes pentes des affluents de l'Aumance et le peu de dépôts conservés dans les vallons. L'encaissement en V est acquis dès le creusement tardiglaciaire, les méandres encaissés observés dans les vallons de la Goutte de l'étang de Saloup et de Morat ont été façonnés par des cours d'eau plus puissants que les ruisseaux actuels. En revanche, le remblaiement sableux des fonds de vallon s'est constitué pour l'essentiel lors des crises érosives générées par des défrichements affectant des secteurs boisés plus ou moins vastes. Des vallons colluviaux en berceau existent entre le début de l'incision et le début de l'écoulement intermittent dans les secteurs cultivés, alors qu'en forêt, l'incision en $V$ et l'écoulement intermittent coïncident le plus souvent. Dans les têtes de vallon et sur les versants, les incisions le plus souvent rectilignes se sont produites pour la plupart en milieu cultivé à la faveur d'abats d'eau exceptionnels, mais elles ne sont conservées que dans les secteurs qui ont été abandonnés par l'agriculture et/ou reboisés. Sous forêt, les formes sont non seulement bien conservées, mais l'érosion continue à se produire après de fortes pluies de saison froide, lorsque les sols sont saturés ou à la faveur d'aménagements mal conçus, comme des fossés de drainage acheminant les eaux directement à l'amont des têtes de ravins. 
JEAN-Pierre LARUE

\section{Remerciements}

Nous remercions M. Bouabdallah (Université Paris XII) pour la réalisation des granulométries, $R$. Étienne (Laboratoire rhodanien de Géomorphologie) pour la détermination des minéraux lourds, F. Bétard (Université Paris XII) pour la mise au net des figures. Cet article a bénéficié des suggestions et critiques constructives de deux relecteurs anonymes.

\section{Bibliographie}

Allée (P.), 2003. - Dynamiques hydrosédimentaires actuelles et holocènes dans les systèmes fluviaux de moyenne montagne (Limousin, hautes Cévennes, Maures), Mémoire d'Habilitation à Diriger des Recherches, Université de Limoges, 451 p.

Allée (P.), Diot (M.-F.), Durieux (J.-F.), Reynet (J.-M.), Valadas (B.), 1997. - « Trois mille ans d'enregistrement sédimentaire dans les fonds de vallons des bas plateaux limousins ", dans La dynamique des paysages protohistoriques, antiques, médiévaux et modernes, $\mathrm{XVII}{ }^{\mathrm{e}}$ Rencontres Internationales d'Archéologie et d'Histoire d'Antibes, Editions APDCA, Sophia Antipolis, p. 365-387.

Allée (P.), Lespez (L.), 2006. - « De l'océanique au méditerranéen, la disparité des réponses morphosédimentaires holocènes, dans les massifs anciens européens ", dans Allée (P.), Lespez (L.) (dir.), L'érosion entre Société, Climat et Paléoenvironnement, Table ronde en l'honneur de R. Neboit-Guilhot, 25-27 mars 2004, Presses universitaires Blaise-Pascal, p. 203-214.

Ambers (R. K. R.), Druckenbrod (D. L.), Ambers (C. P.), 2006. - « Geomorphic response to historical agriculture at Monument Hill », Catena, n 65, p. 49-60.

Bertrand (E.), 1996. Les sites antiques, La lettre du Pays de Tronçais, n 2, p. 1-48.

Blott (S. J.), Croft (D. J.), Pye (K.), Saye (S. E.), Wilson (H. E.), 2004. - « Particle size analysis by laser diffraction », dans Pye (K.), Croft (D. J.), (Eds.), Forensic Geoscience: Principles, Techniques and Applications, Geological Society, London, Special Publication, vol. 232, p. 63-73.

Bridgland (D. R.), Maddy (D.), Bates (M.), 2004. - « River terraces sequences: templates for Quaternary geochronology and marine-terrestrial correlation », Journal of Quaternary Science, vol. 19, n² 2, p. 203 218.

Bull (W. B.), 1991. - Geomorphic responses to climatic change, New York, Oxford University Press, $321 \mathrm{p}$.

Cubizolle H., Valadas B., Gagnaire J., Evin J., 2001. - « La dynamique des versants dans le bassin de la Dore durant la deuxième moitié de l'Holocène (Massif Central, France) : premières données géoarchéologiques et datations radiocarbone », Quaternaire, vol. 12, n 1-2, p. 15-29.

Dambrine (E.), Dupouey (J.-L.), Laüt (L.), Humbert (L.), Thinon (M.), Beaufils (T.), Richard (H.), 2007. - «Present forest biodiversity patterns in France related to former Roman agriculture », Ecology, $\mathrm{n}^{\circ} 88$, p. 1430-1439.

Demoulin (A.), 1998. - « Testing the tectonic significance of some parameters of longitudinal river profiles: the case of the Ardenne (Belgium, NW Europe) », Geomorphology, vol. 24, p. 189-208.

Dietrich (W. E.), Dunne (T.), 1993. - « The channel head », dans Beven (K.), Kirby (M. J.) (dir.), Channel network hydrology, Chichester, Wiley, p. 175-219.

Dotterweich M., 2008. - " The history of soil erosion and fluvial deposits in small catchments of central Europe: Deciphering the long-term interaction between humans and the environment - A review », Geomorphology, vol. 101, p. 192-208.

Horton (R. E.), 1945. - «Erosional development of streams and their drainage basins: hydrophysical approach to quantitative geomorphology », Geological Society of America Bulletin, n 56, p. 275-370.

Jacob (N.), Gob (F.), Bravard (J.-P.), Petit (F.), 2006. - « Les formes fluviales d'une rivière en gorge, le Chassezac (Cévennes, France)», Géomorphologie: relief, processus, environnement, n 1, p. 3-22.

Keller (E. A.), Pinter (N.), 1996. - Active tectonics. Earthquakes, uplift and landscape, Prentice Hall, Upper Saddle River, N.J., 338 p. 
Lach (J.), WrżGa (B.), 2002. - «Channel incision and flow increase of the upper Wisłoka river, southern Poland, subsequent to the reafforestation of its catchment », Earth Surface Processes and Landforms, vol. 27, p. 445-462.

Lang (A.), Bork (H. R.), Mäckel (R.), Preston (N.), Wunderlich (J.), Dikau (R.), 2003. - « Changes in sediment flux and storage within a fluvial system: some examples from the Rhine catchment ", Hydrological Processes, $n^{\circ} 17$, p. 3321-3334.

LANG (A.), MAuz (B.), 2006. - Towards chronologies of gully formation: optical dating of gully fill sediments from Central Europe, Quaternary Science Reviews, vol. 25, p. 2666-2675.

LARUe (J.-P.), Étienne (R.), 1998. - « Les formations détritiques miocènes, pliocènes et quaternaires entre le Massif central et la Sologne (France). Nouveaux éléments d’interprétation », Géologie de la France, $\mathrm{n}^{\circ} 1$, p. 39-56.

Larue (J.-P.), 1999a. - «L'évolution morphodynamique des vallons et vallées dans les bassins de la Maine et du Cher (sud-ouest du Bassin parisien) depuis le Tardiglaciaire », Bulletin de la Société Géologique de France, t. 170, n 6, p. 927-938.

—, 1999b. - «Le Pays de Tronçais (Allier) : valorisation des paysages et déclin démographique, Norois », $\mathrm{n}^{\circ} 183$, p. 431-484.

—, 2008. - «Profils longitudinaux des cours d'eau et tectonique : l'exemple du bassin de l'Aumance (Massif central, France) », Norois, n² 207, p. 71-439.

Liébault (F.), PiÉgay (H.), 2002. - « Causes of 20th century channel narrowing in mountain and piedmont rivers of Southeastern France », Earth Surface Processes and Landforms, vol. 27, p. 425-444.

Liébault (F.), Gomez (B.), Page (M.), Marden (M.), Peacock (D.), Richard (D.), Trotter (C. M.), 2005. - « Land-use change, sediment production and channel response in uplands regions », River Res. Applic., n²1, p. 739-756.

McBride (M.), Hession (W. C.), Rizzo (D. M.), 2008. - «Riparian reforestation and channel change: A case study of two small tributaries to Sleepers River, northeastern Vermont, USA », Geomorphology, vol. 102, p. 445-459.

Menéndez-Duarte (R.), Marquínez (J.), Fernandez-Menéndez (S.), Santos (R.), 2007. - « Incised channels and gully erosion in Northern Iberian Peninsula: Controls and geomorphic setting », Catena, $\mathrm{n}^{\circ} 71$, p. 267-278.

Montgomery (D. R.), Dietrich (W. E.), 1988. - «Where do channels begin? », Nature, n 336, p. 232234.

Montgomery (D. R.), Dietrich (W. E.), 1994. - « Landscape dissection and drainage area-slope thresholds », dans Kirkby (M. J.) (Ed.), Process Models and Theoretical Geomorphology, Wiley, New York, p. 221 246.

Mostey (M. P.), 1974. - « Experimental study of rill erosion », Transactions of the American Society of Agricultural Engineers, $n^{\circ} 17$, p. 909-913.

Parfenoff (A.), Pomerol (C.), Toureng (J.), 1970. - Les minéraux en grains, méthodes d'étude et détermination, Masson, Paris, 578 p.

Pastre (J.-F.), Fontugne (M.), Kuzucuoglu (C.), Leroyer (C.), Limondin-Lozouet (N.), Talon (M.), Tisnerat (N.), 1997. - «L'évolution tardi-et postglaciaire des lits fluviaux au NE de Paris (France). Relations avec les données paléoenvironnementales et l'impact anthropique sur les versants », Géomorphologie: relief, processus, environnement, $\mathrm{n}^{\circ} 4$, p. 291-312.

Pastre (J. F.), Limondin-Lozouet (N.), Leroyer (C.), Ponel (P.), Fontugne (M.), 2003. - « River system evolution and environmental changes during the Late glacial in the Paris Basin (France) », Quaternary Science Reviews, vol. 22, p. 2177-2188.

Phillips (J. D.), Lutz (J. D.), 2008. - « Profile convexities in bedrock and alluvial streams », Geomorphology, vol. 102, p. 554-566.

Prosser (I. P.), Soufi (M.), 1998. - « Controls on channel formation following forest clearing in a humid temperate environment », Water Resources Research, n 34, p. 3661-3671.

Raynal (J.-P.), 1984. - «Chronologie des basses terrasses de l'Allier en Grande Limagne (Puy de Dôme, France) », Bulletin de l'Association française pour l'étude du Quaternaire, n¹-2-3, p. 79-84. 
Rommens (T.), Verstraten (G.), Bogman (P.), Peeters (I.), Poesen (J.), Govers (G.), Van Rompaey (A.), LANG (A.), 2006. - « Holocene alluvial sediment storage in a small river catchment in the loess area of central Belgium », Geomorphology, vol. 77, p. 187-201.

Schumm (S. A.), 1956. - « The evolution of drainage systems and slopes in badlands at Perth Amboy, New Jersey », Bulletin of the Geological Society of America, $\mathrm{n}^{\circ}$ 67, p. 597-646.

Simon (A.), Rinaldi (M.), 2006. - « Disturbance, stream incision, and channel evolution: The roles of excess transport capacity and boundary materials in controlling channel response », Geomorphology, vol. 79, p. 361-383.

SinhA-Roy (S.), 2001. - «A new approach to the analysis of transverse river valley profiles and implications for morphotectonics: A case study in Rajasthan », Current Science, n 81, p. 106-112.

Sklar (L.), Dietrich (W. E.), 1998. - « River longitudinal profiles and bedrock incision models: stream, power and the influence of sediment supply », dans Tinckler (K. J.), Wohl (E. E.) (dir.), River over rocks: fluvial processes in bedrock channels, AGU Geophysical Monograph, n 107, p. 237-260.

Smolska (E.), 2007. - « Development of gullies and sediment fans in last-glacial areas on the example of the Suwałki Lakeland (NE Poland)», Catena, n 71, p. 122-131.

Taylor (S. B.), Kite (J. S.), 2006. - « Comparative geomorphic analysis of surficial deposits at three central Appalachian watersheds: Implications for controls on sediment-transport efficiency », Geomorphology, vol. 78 , p. 22-43.

Turland (M.), Hottin (A.-M.), Cojean (R.), Ducreux (J.-L.), Debeglia (N.), d’Arcy (D.), Mathis (V.), Carroué (J.-P.), Piboule (M.), 1990. - Notice explicative de la feuille d'Hérisson à 1/50000, éd. BRGM, $118 \mathrm{p}$.

Vannière (B.), Laggoun-Defarge (F.), 2002. - « Première contribution à l'étude des évolutions paléohydrologiques et à l'histoire des feux en Champagne berrichonne durant l'Holocène. Le cas du Marais du Grand Chaumet (Indre, France) », dans Bravard (J.-P.), Magny (M.) (dir.), Les fleuves ont une histoire : paléoenvironnements des rivières et des lacs depuis 15000 ans, Édition Errance, p. 101-124.

Vanwalleghem (T.), Van den Eeckhaut (M.), Poesen (J.), Deckers (J.), Nachtergaele (J.), Van Oost (K.), Stenters (C.), 2003. - «Characteristics and controlling factors of old gullies under forest in a temperate humid climate: a case study from the Meerdaal Forest (Central Belgium) », Geomorphology, vol. 56, p. 15-29.

Vanwalleghem (T.), Van den Eeckhaut (M.), Poesen (J.), Govers (G.), Deckers (J.), 2008. - « Spatial analysis of factors controlling the presence of closed depressions and gullies under forest: Application of rare event logistic regression », Geomorphology, vol. 95, p. 504-517.

Wilkinson (K. N.), 2003. - «Colluvial deposits in dry valleys of Southern England as proxy indicators of paleoenvironmental and land-use change », Geoarchaeology, n 19, p. 725-755.

Cet article a été reçu le 6 février 2009 et définitivement accepté le 6 août 2009. 